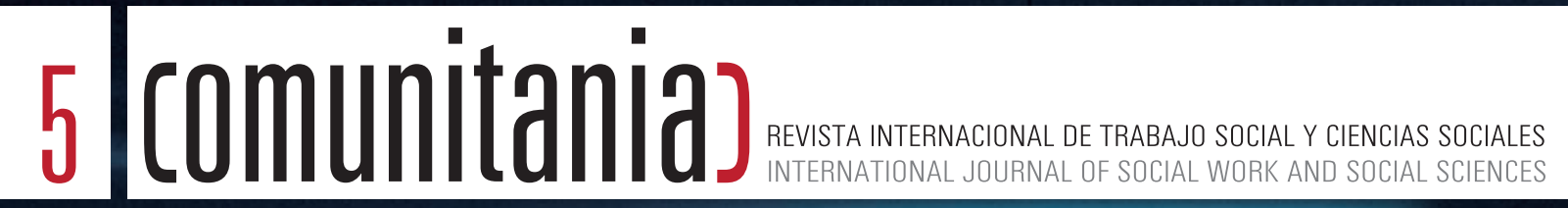

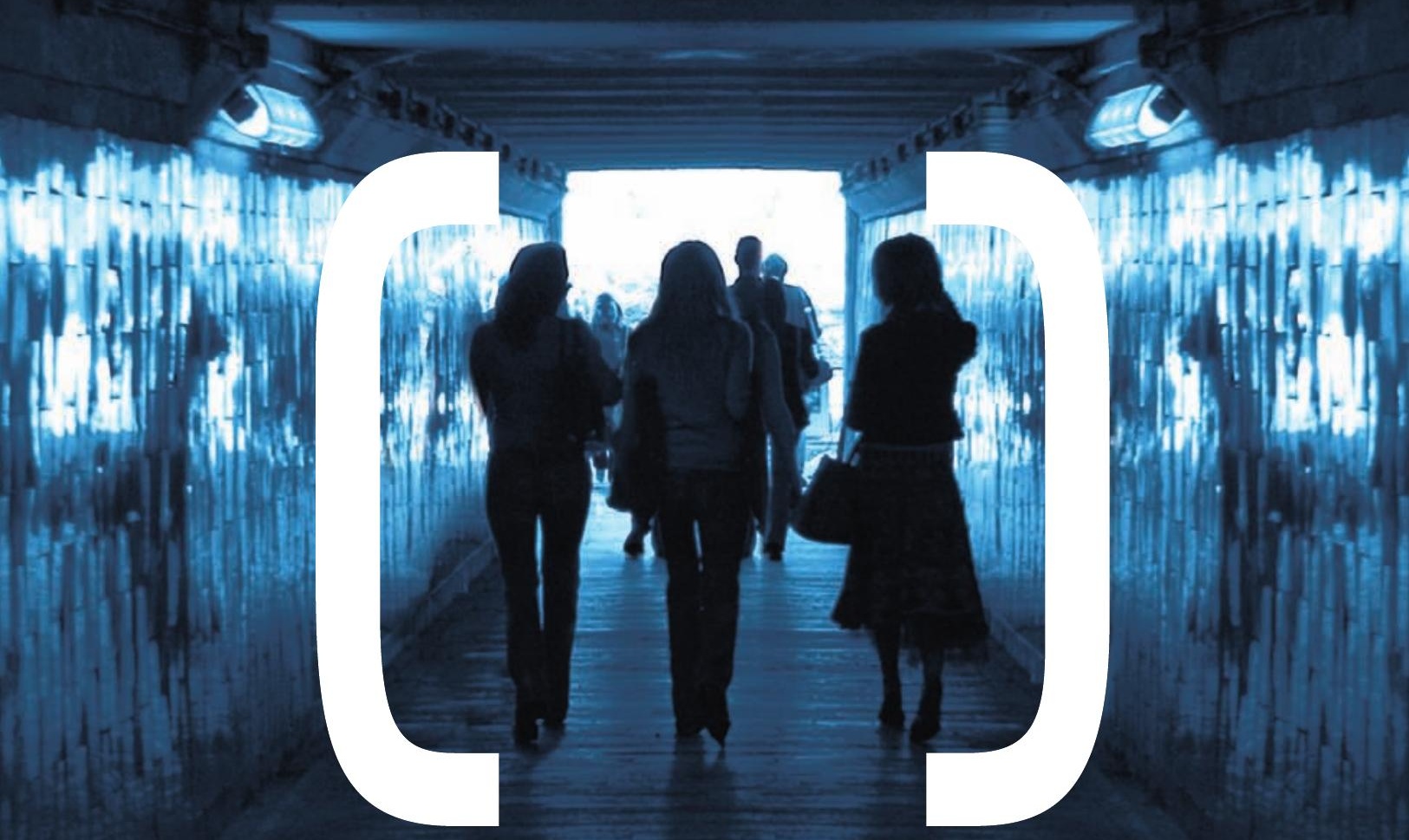

EGUZKI URTEAGA | RODRIGO ANDRÉS | ÁNGELES MARTíNEZ | Ma INMACULADA LÓPEZ | ALEJANDRO CABELLO MARÍA PAZ GARCÍA-LONGORIA | REYNA LIZETH VÁZZUEZ | ARNULFO SÁNCHEZ | EVA SOTOMAYOR | YOLANDA DE LA FUENTE MARTA GARCÍA | Ma LUISA GRANDE | TOMÁS ALBERICH | KARLA SÁENZ | DELIA TÉLLEZ | ANTONIO GUTIÉRREZZ 


\title{
La mediación escolar y las habilidades sociales en los estudiantes de educación secundaría. Un estudio en institutos de la región de Murcia School mediation and social skills students in secondary education. A study in institutes of Murcia
}

\author{
María Paz García-Longoria Serrano* y Reyna Lizeth Vázquez Gutiérrez** \\ * Universidad de Murcia. glongori@um.es \\ ** Universidad Autónoma de Nuevo León. reynavqz@hotmail.com.
}

\begin{abstract}
Abstrac:
We highlight the advantages of mediation in schools as a strategy and tool that benefits school life in basic education, bringing our students to peaceful forms of conflict resolution and social skills that will facilitate their inclusion in the workplace and family. We describe the relationship between the characteristics of a school mediation program and the main objectives set by the peace education from international agencies. As developed in various countries school mediation as a form of holistic education and the main characteristics that define it, also, share the results derived from our field research called: school mediation as a tool for peace education, and present last paragraph of conclusions and proposals. School mediation is considered a multi-impact areas within the school in which to develop.
\end{abstract}

Keywords: Education, Education for Peace, Mediation, Mediation School, Social Skills.

\section{Resumen:}

Destacamos las ventajas de la mediación escolar como estrategia y herramienta que beneficia la convivencia escolar en educación básica, acercando a nuestros estudiantes a las formas pacíficas de resolución de conflictos y el desarrollo de habilidades sociales que facilitarán su inclusión en el mundo laboral y familiar. Exponemos la relación que existe entre las características de un programa de mediación escolar y los principales objetivos establecidos por la educación para la paz, desde los organismos internacionales. Como se ha desarrollado en diversos países la mediación escolar como forma de educación integral y las principales características que la definen, así mismo, compartimos los resultados derivados de nuestra investigación de campo denominada: la mediación escolar como herramienta de educación para la paz, y presentamos finalmente el apartado de conclusiones y propuestas. La mediación escolar, es considerada un programa de múltiples ámbitos de impacto dentro del centro educativo en el que se desarrolle. 
Palabras clave: Educación, Educación para la Paz, Mediación, Mediación Escolar, Habilidades Sociales.

\section{Article info:}

Received: 16/10/2012 / Received in revised form: 05/12/2012

Aceppted: 10/12/2012 / Published online: 20/02/2013

DOI: http://dx.doi.org/10.5944/comunitania.5.6

\section{INTRODUCCIÓN}

Nos encontramos a dos años de la finalización de la denominada Década de la Paz, período comprendido entre el 2001 y el 2010 en el que la Organización de las Naciones Unidas proclamó a los países miembros realizar acciones y estrategias específicas, dirigidas a insertar en los sistemas educativos la formación en valores, el respeto por los demás y con un énfasis importante dedicar las suficiente atención hacia acciones de prevención y disminución de la violencia en las escuelas.

Hablamos de educación para la paz desde un punto de vista integral, pretendemos enaltecer la necesidad a la que nos enfrentamos actualmente, en la que la sociedad a la que pertenecemos cada día se vuelve más exigente en cuanto a la formación de alumnos con miras de futuros ciudadanos y formadores de familias, y no solamente con la percepción de aquellos jóvenes que acuden a la escuela a escuchar al profesor y se van a casa con la lista de deberes para entregar al siguiente día en una rutina igualitaria.

En forma general, se busca principalmente, impulsado por la UNESCO, lograr el objetivo de que los Estados miembros sean apoyados para modificar sus políticas educativas (legislación y práctica) a fin de asegurar que en el programa de estudios se incluyan valores como los derechos humanos, la paz, la participación democrática, la tolerancia, la no violencia y el entendimiento intercultural y, que los proceso educativos vigentes, se ajusten a la enseñanza de dichos valores.

Es importante destacar que en muchos países, la educación forma parte de los principales enfoques de trabajo por parte de los gobiernos por lo que se han dado la constante colaboración entre la instituciones involucradas en busca de realizar las modificaciones e inclusiones curriculares necesarias de acuerdo con el cambio y modernización social.

La educación para la paz, como movimiento internacional es relativamente joven, pero en su corto tiempo de actuación ha podido aportar grandes beneficios educativos y de formación integral a los diversos países involucrados en su desarrollo. 
Pretendemos entender a la educación integral como un todo que se compone de una gran diversidad de elementos, tanto individuales, de recursos materiales y económicos, sociales, familiares, etc. por lo que lo ideal sería lograr el equilibrio en el trabajo en conjunto de todos los elementos que componen a la educación con miras a crear a futuros ciudadanos con habilidades sociales importantes y con el manejo y la interiorización de herramientas comunicacionales que les permitan resolver pacíficamente sus conflictos.

\section{EL CONCEPTO DE EDUCACIÓN PARA LA PAZ Y SUS AVANCES}

Los primeros enfoques de trabajo sobre la EP se ubican a finales de la II Guerra Mundial al igual que los principales trabajos y estudios sobre la paz y la guerra, lo que nos permite afirmar que la $E P$, es una más de las herencias benéficas que nos ha dejado el interés de los primeros teóricos en entender la paz desde las relaciones humanas, en el caso de la educación en España es a finales de la década de los 70, cuando se manifiesta un serio interés por la educación para la paz (Asociación Pro Derechos Humanos 1994).

Se consideran como ejes centrales para el nacimiento de la EP, tanto al movimiento de la noviolencia, dado que, para Gandhi era de suma importancia que la escuela no solo debe ser abierta e integrada en su medio, sino que la educación no puede ser responsabilidad exclusiva de la misma: toda la comunidad debe participar en ella (Jares 1991). Al igual que por la creación de la Liga Internacional de la Escuela Nueva en Europa o su equivalente la Escuela Progresista en EU, aproximadamente a finales del siglo XIX extendiéndose hasta la II Guerra Mundial, consolidada en el Congreso Internacional de Educación Nueva en 1921, alcanzó la implantación en más de veinte países y reúne en sus filas a los pedagogos más prestigiosos del mundo los teóricos dedicados a ella partían de la concepción de que el niño es bueno por naturaleza, por lo que la guerra y la violencia eran cosas de los adultos.

Jean-Jacques Rousseau, Pestalozzi, Froebel y Herbart, quienes son considerados los educadores precursores del movimiento de la Escuela Nueva, partían de la realidad de que no todos los niños son iguales por lo que deben ser tratados de formas diferentes, además de centrar la educación en actividades de cooperación y colectividad, más que en aquellas de individualismo.

Posteriormente, relacionado con la época del nacimiento de la Investigación para la Paz, se reconoce el segundo período de la EP. En realidad podemos decir que la educación para la paz nace desde la influencia de la filosofía ideológica del movimiento de la escuela nueva y los aportes y aperturas brindadas por los estudios generados a raíz de la IP, apoyada también directamente por la escuela moderna de Celestin Freinet, fecha en sus aportaciones de escuelas participativas enfocadas en el papel activo del alumno de educación primaria, la educación centrada en el niño. 
Al término de la guerra, específicamente el 16 de noviembre de 1945 se crea la UNESCO, United Nations Educational, Scientific and Cultural Organization, Organización de las Naciones Unidas para la Educación, la Ciencia y la Cultura, la perspectiva de la organización se centra en el desarrollo del potencial humano, la promoción de los valores universales de libertad, dignidad y justicia, a través de una educación al alcance de todos, del acceso de todos a los progresos de la ciencia y la tecnología, de la valorización del patrimonio cultural y el fomento de la creatividad, de la libre circulación de la información y el desarrollo de la comunicación.

Con el preámbulo de Puesto que las guerras nacen en las mentes de los hombres, es en las mentes de los hombres donde deben erigirse los baluartes de la paz, la constitución de la UNESCO del 16 de noviembre de 1945, expone como objetivos fundamentales en relación a la educación, los siguientes:

- Ampliar el acceso a la educación básica con miras a la educación permanente de todos y renovar su contenido, sus métodos y sus procesos para que se ajusten a las necesidades del desarrollo individual y social.

- Renovar, diversificar y ampliar los sistemas, programas y procesos educativos con miras a la educación permanente y darles más sensibilidad a las transformaciones sociales y a las perspectivas del siglo XXI.

- Fortalecer las capacidades de los Estados Miembros de la Organización a fin de reorientar la educación de todos los niveles hacia un futuro sostenible y promover la toma de conciencia y la formación, así como la cooperación interinstitucional y las actividades conjuntas en este ámbito.

Bajo la influencia de los movimientos mencionados y primordialmente el impulso de los trabajos internacionales por parte de la UNESCO, el enfoque de la educación para la paz, ha logrado cambiar la visión de los conflictos en la educación, para percibirlos actualmente como un proceso natural, producto de las diferencias humanas que se manifiestan en un aula escolar en la que convive un número importante de personas con caracteres distintos.

Para Bover (Jares 1991:36), se configura como un proceso de una triple dimensión; educación moral, educación social y educación religiosa, producto de la crítica pedagógica tradicional por un lado y por otro la necesidad de desarrollar una educación para la comprensión internacional que evite la guerra, es la creación del Bureau International d’Education sin lugar a dudas va a ser decisiva para su divulgación y conceptualización, tanto por el dinamismo desarrollado, como por formar parte del mismo varios de los principales teóricos de la educación para la paz como es el caso del francés Pierre Bover y el catalán Pere Rossello.

Lo cierto es, que no se cuenta con un concepto perfectamente delimitado, Jares (1991) menciona la existencia de tres posibles enfoques hacia su definición: los que propugnan un enfoque negativo, que es el mayoritario y que configura a la educa- 
ción para la paz como contracorriente de opinión en contra de la guerra y el militarismo en general. Los que propugnan un enfoque positivo, pero que en sus recomendaciones prácticas se centran en aquellas de tipo negativo y los que ven la necesidad de combinar las dos perspectivas, como ejemplo, la escuela Montessori, caracterizada por percibir al educando como un ser puro, neutral y libre apartado de todas las ideas filosóficas y partidos políticos.

Bajo la propuesta realizada por la UNESCO en 1980 en el Congreso Mundial de Educación para el Desarme, hablamos de educación para la paz como: un proceso educativo que tiene como objetivo la construcción y emergencia de una opinión ciudadana que rechaza la violencia como forma de resolución de conflictos y que exige cambios cualitativos en la voluntad política (Banda 2002:85), promoviendo la revisión cualitativa de programas, y la inclusión de valores, actitudes y conductas inherentes en una cultura de paz, una formación para la prevención y resolución del conflicto, el diálogo, la creación de consenso y la no-violencia (UNESCO 2002).

La también nombrada pedagogía de la paz, como nos menciona Muñoz (2004) nace como estudio de los comportamientos agresivos y violentos en los seres humanos y apuesta por unas formas de socialización y educación diferentes que hicieran a las personas más libres, responsables y creativas, al día de hoy y revisando los arduos trabajos que se han realizado en busca de esta formación educativa más responsable y participativa, podemos asegurar que la implicación de la escuela en la educación para la paz rinde funcionalidad.

Por otra parte, existen diversas opiniones acerca de la presencia de la educación para la paz en materia de su inclusión en el currículo escolar, por ejemplo Fernández Herrería (1994) opina que, es importante tener en cuenta que la educación para la paz, es también la presencia en la escuela de la opción paz que existe hoy en la sociedad como utopía positiva, que busca respuesta a los graves problemas que hay planteados en nuestras relaciones sociales y que da sentido a la lucha por si la transformación; sin embargo al mismo tiempo destaca que el hablar en sentido amplio de la educación para la paz, no es solo la importancia de un día de la paz que conmemora la muerte de Gandhi, sino todo un planteamiento renovador del funcionamiento global de la institución en que se integran los profesores de Matemáticas, de Lengua, de Sociales, etc. que se hacen todos los profesores también de la tarea de la paz.

En lo que nos corresponde al presente artículo, estamos de acuerdo tanto con Fernández como con lo que nos exponen, por ejemplo, González Valcárcel \& Martínez (1999:33), al hablar de paz en la educación y la formación integral de los educandos lo que pretendemos es evitar dar explicaciones simples a un tema que tiene múltiples facetas y que se manifiesta bajo formas también múltiples dentro del ámbito escolar, al tiempo que nos interesamos por los factores que puedan favorecer la prevención de conflictos no deseables. 
La educación para la paz, cuenta con diversos principios esenciales desde su nacimiento, mismos que han sido alimentados por sus bases de creación más importantes, como las mencionadas anteriormente, sin embargo, es reconocido que en la última década es cuando mayormente se ha visto beneficiada y ha contado con el enriquecimiento en sus bases de acuerdo con los trabajos realizados por los diferentes países colaboradores y las organizaciones interesadas en la educación integral exitosa.

En general, entendemos por educación para la paz, tal como nos menciona Jares (1991:121), un proceso educativo, dinámico, continuo y permanente, fundamentado en los conceptos de paz positiva y en el conflicto como elemento significantes y definidores, y que, a través de la aplicación de enfoques socioafectivos y problematizantes, pretende desarrollar un nuevo tipo de cultura, la cultura de la paz, que ayude a las personas a desvelar críticamente la realidad, para poder situarse ante ella y actuar en consecuencia.

En la comprensión de una educación integral en la que no nos dirigimos a un solo aspecto, sino que por el contrario, resaltamos la necesidad de que la escuela sea formadora de seres humanos capaces de actuar conforme a las herramientas sociales que han adquirido en su larga etapa de educandos.

Somos conscientes de que, debemos todos trabajar en un proceso educativo que signifique contribuir a alejar el peligro de la guerra, poner fin al expolio de las zonas empobrecidas del planeta, enseñar desde y para la noviolencia, aprender a considerar el conflicto como un vehículo de cambio si sabemos resolverlo sin recurrir a la violencia, integrar al alumno en un proceso de transformación de la sociedad hacia la justicia, etc., en definitiva, este va a ser el compromiso de educar para la paz (Asociación Pro Derechos Humanos 1994:14).

Existen diversas formas de integrar la educación para la paz en la escuela, por una parte podría realizarse de manera parcial, es decir, mediante trabajos continuos extracurriculares en el curso, que hagan mención a la importancia de la paz como tema central de las relaciones humanas, elementos basados básicamente en referencias internacionales, días conmemorativos etc. O bien de forma total, en donde se interiorice la necesidad de formas de resolución no violenta de conflictos, actividades de colaboración y cooperación, entre otros elementos que sean insertados como actividades en todas las asignaturas y actividades escolares, y por último de una forma mixta, combinando las dos mencionadas, siendo la más recomendable y la que brinda mayor libertad al centro educativo para las adaptaciones necesarias de acuerdo con sus propias características generales y las de su población estudiantil.

\section{ANTECEDENTES DE LA MEDIACIÓN ESCOLAR}

La mediación escolar como estrategia de resolución de conflictos en los centros 
educativos tiene su nacimiento en las prácticas realizadas en instituciones de los Estados Unidos, se comenzó a trabajar con programas en los que se involucraba a los propios alumnos en un proceso de diálogo y exposición de motivos sobre el problema que se presentará, para posteriormente con ayuda de un tercero, lograr el perdón y la reparación de la relación que existiese.

Según nos cuentan Munné y Mac-Cragh (2006) es en los años 60, que la mediación escolar surge por vía de grupos religiosos y organizaciones para la paz mediante el objetivo de enseñar a los niños resoluciones de conflictos desde la noviolencia, aunque es en los 70 en Estados Unidos de América, específicamente en la ciudad de Nueva York, donde apareció por primera vez un programa de resolución de conflictos dentro de los centros educativos.

Gracias a los resultados satisfactorios del programa, se iniciaron los trabajos para su implementación por todo el país, cuando las escuelas optaron por este tipo de procedimientos pacíficos, utilizaban la mediación para tratar asuntos relacionados con peleas, prejuicios, acoso, comunicación deficiente entre padres e hijos, celos, calumnias, insultos, mal comportamiento en el aula, burlas, amenazas e inclusive robos.

Para la década de los 80, nacen asociaciones como la Educators for Social Responsability, la National Association for Mediation of Education manteniendo diversas formaciones y asociaciones entre todos los interesados en el tema para que finalmente se dé por constituida la Conflict Resolution Education Network, hasta llegar al logro de que en la actualidad el país cuente con más de 5000 programas de mediación escolar, convivencia o educación para la paz.

El éxito se ha podido comprobar también en otros países como Francia, Argentina, Colombia, Nueva Zelanda, Inglaterra, Canadá, Chile, Costa Rica, entre otros que aun cuentan con la mediación escolar como programas iniciales de convivencia y de estrategias de disminución de conflictos.

En Argentina, por ejemplo, Florencia Brandoni en su informe denominado Una introducción a la mediación escolar, que presenta para los trabajos de investigación de la Red Diálogos Productivos, nos habla de que es en la última década cuando más se ha mostrado una gran preocupación oficial por este tema, y, que actualmente se encuentran en marcha varios programas de mediación escolar, cuyos fundamentos son la búsqueda de la disminución de la violencia. Lideran esta política pública el Ministerio de Justicia y el Ministerio de Educación de la Nación.

La experiencia en Argentina, da inicio de manera formal y profesional desde 1996, tal es el éxito y la participación de numerosas asociaciones que actualmente Buenos Aires y las provincias de Chaco, Misiones y Rio Negro cuentan con una Ley de Mediación Escolar que regula la aplicación de los programas y la participación de las asociaciones a cargo del Ministerio de Educación, con fundaciones y organizaciones 
activas como, Fundación Alternativa, Gobierno de la Ciudad de Buenos Aires, Ministerio de Justicia, Provincia de Chaco, Asociación Virgen de la Paz y el Ministerio de Educación de la Nación.

En Venezuela, también ha tomado un gran auge la aplicación de programas de mediación escolar, en el caso de este país se comenzó unos años después de la experiencia de Argentina, y su aplicación inicial fue dirigida a programas en pro de la disminución del bullying, el éxito que han tenido los programas iniciales ha permitido su extensión y la participación de asociaciones interesadas en la educación para la paz.

En Europa, por su parte a lo largo de los últimos 25 años el estudio de los conflictos en la escuela ha sido una cuestión prioritaria. Este hecho ha generado diversas formas de tratamiento de estos conflictos, con resultados ampliamente efectivos (Munné y Mac-Cragh 2006:19). Actualmente participan en programas de convivencia escolar por la línea de la mediación países como Inglaterra, Francia, Italia, etc. la segunda de ellas con un gran avance respecto a la mediación escolar en nivel de preescolar.

De esta diversificación, en el terreno de la mediación se hace eco el ámbito educativo en España, incorporando por un lado, los programas de mediación escolar, desde la perspectiva de la resolución de conflictos, y por otro la mediación social intercultural trabajando en la escuela desde la perspectiva de la convivencia intercultural (Pérez Crespo 2002).

Es en 1993, cuando gracias a la iniciativa del Centro de Investigación para la Paz Gernika Gogoratuz del País Vasco, se pone en marcha el primer programa piloto de la mediación en las escuelas, extendiéndose rápidamente por el resto de comunidades españolas, en 1996 inician los trabajos en Catalunya, donde actualmente su éxito ha permitido que todos los Institutos de Educación Secundaria cuenten con un programa de mediación escolar. Ese mismo año, Canarias realiza su Proyecto Educativo para la mejora de la convivencia. En 1998 la Comunidad de Madrid lanza el programa piloto que posteriormente se extiende, gracias a los resultados, a una gran parte de sus colegios e Institutos, en 2003, Andalucía trabaja en el programa denominado Escuelas: Espacio para la Paz y desde 2004 la Comunidad Valenciana lleva a cabo encuentros y jornadas de trabajos sobre la mediación escolar.

Hoy en día, es cada vez más creciente el interés de diversos países en la participación de programas de mediación escolar, iniciando con proyectos pilotos y posteriormente implementando los mismos a los institutos, países como Polonia, Alemania, Sudáfrica, se han sumado a la incorporación de estrategias de convivencia y solución pacifica de los conflictos escolares.

En el caso de los países Nórdicos, se utiliza desde el 2006 financiado por el Ministerio de Educación, un programa denominado La Pirámide del Conflicto (Hareide 
2006), en el que al surgir un conflicto entre estudiantes, estos pueden tomar la decisión de resolverlo por sí mismos mediante el diálogo y la otorgación del perdón y en caso de que esto no funcione, podrán acudir ante un mediador que puede ser alumno o profesor, y en el caso de que la mediación no funcione, entonces será impuesta una sanción por un superior.

De las diversas experiencias que se han aplicado en países, tanto en América como en Europa, se han podido establecer características principales de los programas de mediación escolar.

\section{CARACTERÍSTICAS Y ALCANCES DE LA MEDIACIÓN ESCOLAR}

La mediación escolar, es el proceso de diálogo para la resolución pacífica de conflictos por el cual, las partes enfrentadas encuentran soluciones consensuadas con la intervención de una tercera persona neutral el mediador, que ayuda, facilitando el entendimiento entre las partes, a que estas soluciones sean satisfactorias y restablezcan la convivencia en las aulas (García-Longoria y Ortuño Muñoz 2010).

En el ámbito de la convivencia escolar, son diversas las acciones a las que se recurre, dentro de cada uno de los centros, los directores y los maestros planean y aplican estrategias, técnicas y programas en busca de la mejora de la convivencia, desde nuestra opinión, es importante que el centro cuente con la oportunidad de realizan un diagnóstico general previo y determine las estrategias en base a los resultados del mismo. Joan Teixidó (2010) destaca la importancia de establecer un sistema de coordenadas que le permita formarse una visión global del yacimiento.

Ángel Calvo Rodríguez (2003), por su parte, nos destaca, que son diversos los caminos hacia donde un centro educativo puede dirigir sus trabajos, para la mejora de la convivencia escolar y expone las siguientes estrategias de adaptación:

- Adaptación a las variables personales del alumno. La atención orientadora se dirige fundamentalmente a valorar su capacidad intelectual ya que ésta determina en gran medida la posibilidad de aprender.

- Adaptación a las variables sociofamiliares del alumno. Cuando la familia valora el trabajo escolar se facilita el progreso académico, especialmente si esta valoración va acompañada de conductas que favorecen su aprendizaje.

- Adaptación a las tareas de aprendizaje. Actividades relacionadas con los intereses y experiencias del alumno, que vayan en secuencia, que se propongan las tareas como actividades de aprendizaje para aplicación diaria, que se demuestre la utilidad de estos aprendizajes en el entorno próximo del alumno. 
- Atención a las relaciones que se establecen en el aula. La reacción del alumno ante la escuela también está influida por las interacciones que tiene con el profesor y con sus compañeros.

La adaptación de las estrategias, los programas y las actividades para la mejora de la convivencia, son responsabilidad del centro educativo, en base a su funcionamiento y análisis general de la situación, sin embargo, la mediación escolar, puede considerarse una herramienta moldeable, que tiene le eficacia de impactar en todos los ámbitos cercanos al alumno, en beneficio de su comportamiento.

La conflictividad escolar, puede traer como consecuencias negativas, la disminución del rendimiento escolar, modificación y degradación del rol del profesor, Fernández Herrería (1994), nos explica también, que podemos enfrentarnos a la existencia de: conflictos visibles y conflictos invisibles en el centro educativo, los primeros se identifican como, aquellos conflictos que causan una alteración al ritmo del aula o del centro educativo, y los segundos, son aquellos que afectan frecuentemente a necesidades de los alumnos, que no se manifiestan con expresiones que atenten contra las necesidades institucionales, es decir, aquellos problemas entre los alumnos, que se podrían esconder detrás de situaciones cotidianas.

La mediación hace frente a los conflictos admitiéndolos, explorándolos y considerándolos oportunidades de aprendizaje (Puig 1997 en Boqué Torremorell M. C. 2010), al mismo tiempo que fortalece a las personas, les da voz y las hace protagonistas de su vida (Bush y Folger 1996). Para lograr que los conflictos educativos se conviertan en oportunidades de aprendizaje, tanto para el centro, como para los alumnos que participan en el conflicto, es necesario orientar las estrategias de intervención correctamente y bajo un pleno conocimiento de las condiciones generales que se manifiestan en el ambiente escolar diario del centro.

Sobre la necesidad de un diagnóstico o estudio general de la convivencia, y la participación de los maestros en el centro educativo, diversos autores han expuesto su opinión, acerca de las opciones que se tiene como centro educativo, para dirigir las estrategias en la mejora de la convivencia.

Torrego y Moreno (2003) establecen, por ejemplo, 10 ámbitos de actuación que consideran más importantes de identificar, y que ayudan para encaminar acertadamente los programas de convivencia o de mediación escolar: el conocimiento del alumnado, los cambios en el currículum, las normas de comportamiento en el aula, la colaboración con las familias, el entorno social del alumnado, la mejora de los procesos de gestión en el aula, las habilidades de comunicación y resolución de conflictos, las medidas organizativas, las normas de convivencia en el centro y las condiciones mínimas de seguridad

Otro enfoque nos dice, que los ámbitos esenciales para la mejora de la convivencia escolar son (Teixidó Saballs 2010): la dimensión educativa, la dimensión profesional, la dimensión comunitaria, la dimensión organizativa y la dimensión operativa. 
Por su parte, Xesús Jares (2006), destaca, seis marcos de convivencia en los que se debe centrar el impacto de una estructura creada para la disminución de conflictos escolares: la familia, el sistema educativo, el grupo de iguales, los medio de comunicación, los espacios e instrumentos de ocio y el contexto político, económico y cultural dominante.

Desde nuestro enfoque, apoyamos la idea de un diagnóstico general previo al establecimiento del programa de mediación escolar como programa educativo para la mejora de la convivencia y como herramienta práctica para fortalecer la educación para la paz en los alumnos de educación secundaria. Creemos que, al ser precedida por un estudio general de centro educativo, su aplicación partirá del conocimiento de la cooperación de profesores, los tipos de conflictos más frecuentes, la identificación del contexto social y familiar de los miembros de la comunidad educativa, y otros elementos esenciales, que ayuden al éxito del programa.

La mediación escolar, como programa para la prevención y la resolución de conflictos dentro de la escuela, es reconocida, como un enfoque de educación para la paz que pretende un nueva imagen de los conflictos y el aprendizaje de las técnicas de análisis y regulación de conflictos de modo noviolento (Fernández Herrería 1994). El aprendizaje de técnicas y el apego a los principios fundamentales de la mediación, desarrollan en el adolescente.

Cuenta con diversas características, que la distinguen de los demás programas de convivencia educativa, en primer lugar, impacta directamente con la participación activa del alumnado, del profesorado, y de todos los miembros del centro educativo, además de ser un programa que crece continuamente y con ello asegura, a largo plazo, el éxito de participación.

Para Aparicio (2002) en (Pérez-Fuentes et al. 2010), por ejemplo, las principales características de la mediación son:

- Sistema en el que intervienen dos o más sujetos conscientes de sufrir un conflicto que no pueden pero que desean resolver y recurren a una tercera persona neutral, cuyo objetivo es establecer la comunicación y velar por los intereses de ambas partes.

- Modelo integrado de resolución de conflictos.

- Proceso alternativo y no opuesto a otras vías resolutivas de conflictos del centro educativo.

- Participación voluntaria por las partes implicadas ya que son éstas las que solicitan el proceso de mediación. 
- Actitud cooperativa y negociadora por ambas partes, de manera que la búsqueda de una solución satisfactoria sea fácil.

- Mediador imparcial a ambas partes, con habilidades sociales y comunicativas.

González Valcárcel \& Martínez (2007:38), nos sugieren, citando a Pearson (1984), algunas orientaciones a las que se pueden dirigir acciones de intervención a favor de la mejora de la convivencia en el centro educativo, desde nuestra opinión, concuerdan con lo que reúne como características esenciales la mediación escolar: crear un ambiente en el que la cooperación, la comunicación, la autodiscusión y la conciencia de grupo se desarrolle; canalizar la desorganización hacia una conducta constructiva; conseguir que los alumnos participen en la solución de los problemas de los demás, a la vez que se comprometan a establecer su propia función social; reforzar los valores positivos de cada uno; organizar discusiones productivas en clase y evitar que los conflictos se agraven.

Con lo anterior, nos damos cuenta de que la mediación escolar no solo se debe implementar como una estrategia aislada de resolución de conflictos, sino que su éxito dependerá de la implicación de todos los relacionados con el centro en la búsqueda de un cambio significativo de actitudes y reacciones ante el conflicto, para lograr aprender de cada situación que se presente. Hay que integrar dinámicas profundas de la institución con las actividades docentes y ser consecuente con lo que ello pueda plantear (Fernández Herrería 1994).

La participación de los adolescentes en programas que requieran cambios de actitudes, más que solo llevar a cabo actividades que exige el propio programa, permite a los alumnos encaminarse hacia la adquisición de habilidades que le beneficiarán en un futuro para aprender a detectar en sí mismos el enfado o la ansiedad cuando se enfrenten a un conflicto y lograr corregir los pensamientos y controlar las reacciones (Beck 2003).

\section{EL DESARROLLO DE HABILIDADES SOCIALES Y LA MEDIACIÓN ESCOLAR}

La principal ventaja de la mediación escolar la encontramos en su naturaleza de proceso educativo, resiste más el paso del tiempo y facilita la comunicación, el diálogo, la escucha, la mediación es una negociación asistida, si la escuela enseña a negociar asistidamente está haciendo un trabajo muy educativo (García-Longoria \& Ortuño Muñoz, 2010). Villaescusa Alejo (2010), considera, que la mediación escolar es un procedimiento tanto de prevención de conflictos como de resolución de los mismos, potencia y transmite los valores del diálogo, la cooperación y el respeto; contribuye al desarrollo personal y social al poner.

Una de las principales ventajas que los adolescentes participen en un programa, como el de la mediación escolar, es que se les permite desarrollar habilidades socia- 
les importantes para sus relaciones cotidianas, dentro y fuera del centro educativo. Significará, para los mediadores, salir del silencio y la pasividad para comprometerse voluntariamente en los procesos de cambio y mejora por el camino del diálogo, el respeto, la cordialidad, la creatividad, la cooperación y el acuerdo (Boqué Torremorell M. C. 2010).

El aprendizaje del procedimiento es en sí mismo positivo, ya que, el alumno, habrá adquirido una serie de habilidades y estrategias que le serán útiles en sus relaciones interpersonales, colaborando en su desarrollo personal y social (Villaescusa Alejo 2010). Son diversas las habilidades que se logran desarrollar, tanto en los mediadores, como en los alumnos que acuden ante ellos para la resolución de conflictos, entre las más importantes para su futura inclusión laboral y familiar destacan, la empatía, el autocontrol, la asertividad y la escucha activa.

Tal como es el caso de los mediadores profesionales, los escolares adoptan su propio estilo de mediador, y con ello el desarrollo de una u otra técnica con mayor soltura, una vez que se encuentran apegados al programa y les agrada su papel de ayudar a los demás a solucionar conflictos, adquieren su propio ritmo en la utilización de técnicas, la mediación escolar debe respetar en todo momento su característica de flexibilidad.

A continuación, describimos algunas de las habilidades que, desde nuestra opinión, son las que más ayudan al alumno, en sus futuras relaciones sociales.

\section{a) La Empatía}

La empatía, es la capacidad de ponerte en el lugar del otro para entender su forma de visualizar las cosas, en este caso, el conflicto, para los alumnos en la edad de la adolescencia, es una de las habilidades más difíciles de desarrollar y aplicar cotidianamente, ya que en esa etapa del desarrollo nos encontramos en un comportamiento egocéntrico, que nos permite entendernos más a nosotros mismos que a los demás. Hasta en el caso de los adultos, cuando nos enfrentamos a conflictos personales cotidianos, generalmente lo primero que utilizamos como recurso es la confrontación (Lederach 1984).

Manuel Segura (2005), utiliza el término de inteligencia interpersonal, basado en la teoría de las inteligencias múltiples de Howard Gardner (psicólogo e investigador de la Universidad de Harvard, experto en el análisis de las capacidades cognitivas), para hacer referencia a la capacidad de ponerse en el lugar de los otros y relacionarse bien con ellos, saber comprender a los demás, saber ayudarles a controlarse, saber motivarlos cuando no lo están, la mediación escolar pretende que los alumnos desarrollen estas habilidades para aplicarlas a sus relaciones sociales y familiares. 
La empatía, se convierte en una de las herramientas esenciales para los mediadores escolares y una de las más apreciadas para los alumnos que acuden ante los mediadores a solucionar un conflicto, el desarrollar la capacidad de entender a los demás y su forma de pensar, permite que la comunidad haga eco de las ventajas de la solución de problemas por vía del diálogo y el acuerdo.

\section{b) La Asertividad}

La habilidad de saber expresarte correctamente ante cualquier situación social o conflictiva que atravieses, es una de las más complicadas de adoptar a lo largo de nuestro desarrollo de la personalidad, comúnmente somos influenciados por los códigos comunicacionales de nuestro entorno cercano, por lo que la asertividad en ocasiones queda en segundo plano.

La asertividad, destaca como el centro y meta de todas las habilidades sociales, ser asertivo quiere decir ser justo y eficaz en la relación interpersonal, siendo asertivo se consigue no solo el éxito social, sino también la satisfacción personal profunda (Segura 2005). La habilidad de ser asertivos, hace referencia la expresión de los propios sentimientos, ya sea positivos o negativos, de forma clara, correcta y que no ofenda a los demás (Cano et al. 2009).

La asertividad, es una de las habilidades sociales que se busca desarrollar con la mediación escolar en los alumnos, mediante el respeto de los tiempos de exposición, la escucha con atención, las preguntas y la búsqueda de información, los mediadores consiguen que los alumnos que acuden a mediación logren expresarse correcta y pausadamente, atendiendo a las necesidades de la otra persona y no solamente a las de ellos mismos.

\section{c) La Escucha Activa}

Los mediadores escolares, aprenden y utilizan en cada momento, desde su formación hasta las sesiones de mediación en las que participan, la habilidad de escuchar activamente a sus compañeros. Ellos se preparan como mediadores escolares en conciencia de que hay que escuchar de las partes, sus necesidades y sus prioridades, sin imponerles ideas propias (Fisas 2002).

Escuchar con atención, brinda ventaja a los mediadores para la identificación de las verdaderas necesidades y sentimientos de sus compañeros en conflicto, entenderlos y permitir que se comprendan entre ellos, al igual que permite en los mediados percibir un ambiente donde se le brinda confianza y atención, asegurando la apertura de la comunicación. Cuando escucho realmente a una persona, incluido el significado importante para ella en aquel momento, oyendo no solo a las palabras, sino a la persona en sí y cuando le hago saber que he captado su propio significado privado, se abre al proceso del cambio (Rogers 1980). 
La forma activa de escuchar es necesaria para entender las necesidades y sentimientos de la otra persona (García-Longoria y Sanches Urios 2004). Además, permite desarrollar diversas técnicas comunicacionales que le benefician al mediador y a los mediados en sus relaciones cotidianas con los entornos sociales más cercanos. Torrego (2000) nos menciona algunas de éstas técnicas como el mostrar interés, clarificar la información, parafrasear lo escuchado, reflejar la exposición y los sentimientos de los demás y resumir correctamente la información extrayendo solo la visión objetiva del conflicto.

La adquisición y el uso de técnicas y herramientas que se relacionan con la escucha activa, no solo beneficia al propio alumno mediador, sino también a aquellos que acuden a resolver conflictos entre iguales, ya que, mediante la guía de un tercero, aprenden a respetar tiempos de habla, a reconocer emociones en los demás, entenderlas y ampliar su perspectiva sobre las opciones de solución. Al igual queTorrego (2000), son muchos los autores que están de acuerdo en que la resolución pacífica de los conflictos educativos por mediación, ayuda a la formación integral en los alumnos.

Tal es el caso del Programa Taldeka, impulsado por el grupo GEUZ y la Comunidad Autónoma del País Vasco, en el que nos afirman que la base de las actividades propuestas son sobre los valores de la escucha, el diálogo, la comunicación efectiva, el respeto al otro, la participación democrática, el aprendizaje experiencial y la inteligencia emocional (De la Herrán Gascón 2010). En este programa, se nos muestra una propuesta de actuación, sobre la prevención y la intervención de conflictos educativos, en busca de la mejora de la convivencia escolar en los centros educativos del País Vasco y extiende sus efectos, hacia los tres principales agentes sociales que influyen en la formación de los educandos, por lo que propone actividades, tanto para profesores, alumnos, familias y sociedad. Todas ellas encaminadas al desarrollo y fortalecimiento de las habilidades sociales en los alumnos.

\section{DISEÑO DE INVESTIGACIÓN}

\section{Tipo de Estudio}

El estudio ha sido realizado siguiendo un modelo de investigación cuantitativo de alcance explicativo, dirigido a alumnos de educación secundaria en donde el principal objetivo se centra en la identificación de habilidades sociales que desarrollan los alumnos mediadores en comparación con aquellos estudiantes que no han tenido contacto alguno con programas de convivencia y la explicación de la relación entre los resultados obtenidos.

\section{Institutos Participantes}

En la Región de Murcia, se ha optado por dar participación a tres IES que actualmente aplican el programa de Mediación Escolar dentro de su funcionamiento dia- 
rio de programas de convivencia. La elección de estos tres institutos participantes se realizó con la intención de abarcar poblaciones educativas de diversas características tanto sociales como económicas y culturales, y se eligieron a los participantes en programas de mediación escolar con el fin de comparar opiniones entre los alumnos de la ESO de la población general con los alumnos que ejercen la función de mediadores escolares. Describimos a continuación las principales características de los tres IES de la Región de Murcia:

Instituto de Educación Secundaria IES Aljada que se encuentra ubicado en calle Ermita Vieja, número 26, 30006, Puente Tocinos, Murcia. Los alumnos se caracterizan principalmente por provenir de familias compuestas por dos o tres hijos en las que en los últimos cinco años se ha dado el crecimiento del factor de ambos cónyuges trabajando, la pedanía ha sido afectada también en este período por el paro en crecimiento general en España, sin embargo, aún no se han dado cifras excedentes dentro de esta población. El mismo centro reconoce que en lo referente a la ubicación del nivel cultural educativo de los padres de familia, se puede dar ubicación en el nivel medio-bajo, mayoritariamente contando solo con los estudios de educación primaria. Por último el centro ha ido experimentando de igual forma el crecimiento en la inclusión de alumnos procedentes de padres inmigrantes, sin embargo, existen muy bien delimitados los programas para la integración y no se ha generado mayor conflicto con ello, también se presenta el factor de nuevas familias con padres jóvenes, de lo que se benefician por su interés en involucrarse en las actividades del IES a favor del éxito escolar.

Instituto de Educación Secundaria Licenciado Francisco Cascales ubicado en Avenida Teniente Flomesta s/n 30001 Murcia, Murcia. Ubicado en el caso antiguo de la ciudad de Murcia, por lo que se caracteriza por recibir a los hijos de las familias que habitan en los alrededores del centro histórico de la ciudad. Por la ubicación y la representación en la historia que caracteriza al IES Cascales su población estudiantil es mayoritariamente proveniente de familias de clase media y media-alta, en la que se mezclan tanto padres con un cónyuge trabajador como con ambos trabajando y principalmente formadores de familias con 2-3 y 4 hijos.

Instituto de Educación Secundaria Villa de Alguazas ubicado en Calle América $s / n$ 30560, Alguazas, Murcia. Ubicado en un municipio de la Región de Murcia dentro del núcleo de la Huerta Murciana y donde su principal actividad hasta hace unos años era la propia agricultura, su población es pequeña. Es un centro con una población estudiantil menor a la de los IES mencionados anteriormente y es un centro educativo en el que sus profesores y directivos han delimitado muy claramente sus acciones en programas específicos tanto de aprendizaje como de convivencia e integración lo que le da un funcionamiento exitoso en general. 


\section{Muestra}

Los alumnos participantes han sido divididos en dos grupos.

Por una parte, nos interesa conocer sobre las habilidades sociales que los estudiantes utilizan en caso de conflicto, desde la perspectiva de los alumnos que representan a la generalidad de la población estudiantil de educación secundaria. Para la toma de esta muestra hemos debido realizar diversas adaptaciones en tiempos, horarios y disponibilidad de clases para llevar a cabo la aplicación, hemos utilizado un modelo de Muestreo por Racimos, conociendo la población dividida por grados de la ESO se ha intentado recoger las opiniones de por lo menos un grupo de cada uno de los grados siguiendo a (Glass \& Stanley, 1994). Hemos logrado contar con la participación de un total de 92 alumnos.

$Y$, por otra parte, alumnos que ya realizan mediaciones dentro de sus programas en los centros educativos. En este caso, hemos encuestado al universo de la muestra, un total de 35 alumnos mediadores, de los cuales 13 son estudiantes del IES Aljada, 16 del IES Cascales y 6 del IES Alguazas.

\section{Instrumento}

Como técnica de recogida de información se utilizó un cuestionario de preguntas cerradas. Éste se construyó en diversas etapas, iniciamos con la consulta de fuentes relacionadas con los programas ya existentes de convivencia escolar, una de las que principalmente retomamos su lenguaje y organización es el Cuestionario para Estudiantes sobre el Proceso de Intervención en Conflicto y Violencia (Ortega 2008).

Se elaboró en primer momento un cuestionario piloto compuesto de 29 ítems con respuestas de escala tipo Likert de 4 puntos donde A es siempre, B es casi siempre, C es pocas veces y D es Nunca, y con el objeto de realizar la construcción de un instrumento adecuado y correctamente redactado en relación a la población estudiantil a la que iba dirigido, se solicitó la retroalimentación de profesores de la ESO, para posteriormente realizar la aplicación piloto, en ella participaron siete alumnos miembros del programa de Mediación Escolar, con el objetivo de comprobar la familiarización con el lenguaje de los ítems, la comprensión sobre la redacción de los mismos y la disponibilidad de los alumnos al número de ítems del instrumento. De la aplicación de la prueba piloto y las opiniones de los docentes, se realizaron las modificaciones pertinentes, dando como resultado final un cuestionario compuesto de 22 ítems cuyas unidades de análisis se describen en la Tabla 1. 
TABLA 1. Unidades de Análisis. Cuestionario Alumnos ${ }^{1}$

\begin{tabular}{|l|c|}
\hline \multicolumn{2}{|c|}{ AGRUPACIÓN DE ÍTEMS PARA ESTUDIO DE VARIABLES } \\
\hline \multicolumn{1}{|c|}{ VARIABLE } & ÍTEMS DEL CUESTIONARIO \\
\hline FAC= Formas de Afrontar los Conflictos & $9,10,11,12,19$ \\
\hline TC=Tipos de Conflictos & $1,3,4,5,7,16$ \\
\hline FR= Formas de Resolución de Conflictos & $6,13,14$ \\
\hline $\begin{array}{l}\text { CPC= Conocer el Programa de Mediación } \\
\text { Escolar o Convivencia }\end{array}$ & $2,8,15,17,18,20,21,22$ \\
\hline
\end{tabular}

\section{ANÁLISIS DE RESULTADOS}

Se ha realizado el análisis de datos mediante el programa estadístico SPSS, en donde analizado los resultados obtenidos sobre las formas más frecuentes que los alumnos de educación secundaria utilizan para afrontar sus conflictos con otros compañeros:

1. Reaccionar ante un conflicto gritando o alzando la voz,

2. Pedir ayuda a otra persona cuando se encuentran en algún conflicto,

3. Ignorar por completo al compañero con el que tienen el conflicto,

4. Intentar entender el punto de vista del compañero,

5. O bien, lograr entender la perspectiva del otro respecto al problema que se desarrolla.

Del análisis estadístico de contraste mediante la Prueba Kruskal-Wallis encontramos diferencias significativas en relación a lo anterior, entre el grupo de los alumnos mediadores y los alumnos no mediadores ajenos al programa (Véase Tabla 2). Específicamente, esta prueba nos arroja que en las dos respuestas relacionadas con conductas empáticas es donde se localizan porcentajes de diferencia significativa, por lo que acudiremos ahora al análisis de frecuencia para describir dichas observaciones.

Es de destacarse que, en el caso de los ALUMNOS que NO son MEDIADORES manifiestan una mayor incidencia a reaccionar frente a un conflicto con formas relacionadas con la evitación. Este grupo de alumnos en un $75 \%$ de las respuestas manifestó tendencia a elegir ignorar al compañero con el que se enfrentan en un conflicto, por delante de opciones como tratar de entenderlo.

\footnotetext{
1 Vázquez Gutiérrez, Reyna L. La Mediación Escolar como Herramienta de Educación para la Paz. Tesis Doctoral. Universidad de Murcia. Julio 2012. Tabla 10. Agrupación de ítems. Cuestionario Alumnos. Pág. 250.
} 


\section{TABLA 2. Estadísticos de Contraste Prueba Kruskal-Wallis sobre Las Formas de Afrontar los Conflictos Agrupación Mediador y no Mediador ${ }^{2}$ Estadísticos de contraste ${ }^{a, b}$}

\begin{tabular}{|c|c|c|c|c|c|}
\hline & $\begin{array}{c}\text { Cuando un } \\
\text { compañero } \\
\text { trata de } \\
\text { imponer sus } \\
\text { ideas, gritas e } \\
\text { insultas }\end{array}$ & $\begin{array}{l}\text { Cuando un } \\
\text { compañero } \\
\text { trata de } \\
\text { imponer sus } \\
\text { ideas, pides } \\
\text { ayuda }\end{array}$ & $\begin{array}{l}\text { Cuando un } \\
\text { compañero } \\
\text { trata de } \\
\text { imponer sus } \\
\text { ideas, lo } \\
\text { ignoras }\end{array}$ & $\begin{array}{l}\text { Cuando } \\
\text { tienes } \\
\text { problemas } \\
\text { con algún } \\
\text { compañero } \\
\text { tratas de } \\
\text { entenderlo }\end{array}$ & $\begin{array}{c}\text { Has } \\
\text { entendido la } \\
\text { manera de } \\
\text { pensar en } \\
\text { algún } \\
\text { compañero } \\
\text { con el que } \\
\text { tengas algún } \\
\text { problema }\end{array}$ \\
\hline Chi-cuadrado & 2,885 & ,010 & 2,619 & 14,144 & 5,946 \\
\hline $\mathrm{Gl}$ & 1 & 1 & 1 & 1 & 1 \\
\hline Sig. Asintót. & ,089 & ,919 & , 106 &, 000 & ,015 \\
\hline
\end{tabular}

a. Prueba de Kruskal-Wallis b. Variable de agrupación: Alumno Mediador o No Mediador

Sin embargo, respecto al grupo de ALUMNOS MEDIADORES encontramos que para ellos las formas más frecuentes mediante las que resuelven los conflictos con sus compañeros son la de tratando de entender al otro, o bien, destacan que han logrado en ocasiones comprender el punto de vista de su compañero cuando han participado en algún conflicto. Mediante el análisis de frecuencia en las respuestas de este grupo encontramos que estas dos principales opciones abarcan un $64 \%$ de sus respuestas, es decir, es notorio que mayoritariamente el grupo de alumnos mediadores utilice formas empáticas para resolver los conflictos que han tenido con alguno de sus compañeros.

Respecto a esto, los resultados obtenidos nos permiten destacar que los alumnos que ya están involucrados con el programa como mediadores escolares, manifiestan sus respuestas ante el conflicto desde la empatía, es decir, intentan entender y comprender a sus compañeros cuando están ante un conflicto; $y$, en el caso de los alumnos que nos son mediadores, ni se han relacionado con el programa en su centro, manifiestan la tendencia a ignorar antes que las relacionadas con la empatía, es decir, podría ser más frecuente que los alumnos intenten evitar el conflicto, con lo que no estarían desarrollando habilidades sociales útiles para su futura inclusión social, familiar y laboral.

2 Vázquez Gutiérrez, Reyna L. La Mediación Escolar como Herramienta de Educación para la Paz. Tesis Doctoral. Universidad de Murcia. Julio 2012. Tabla 52. Estadísticos de Contraste Prueba Kruskal-Wallis sobre Las Formas de Afrontar los Conflictos Agrupación Mediador y no Mediador. Pág. 301. 


\section{GRÁfICA 1. Frecuencia sobre las Formas de Afrontar los Conflictos Agrupación Mediadores y No Mediadores ${ }^{3}$}

FORMAS DE AFRONTAR LOS CONFLICTOS-MEDIADORESY NO MEDIADORES

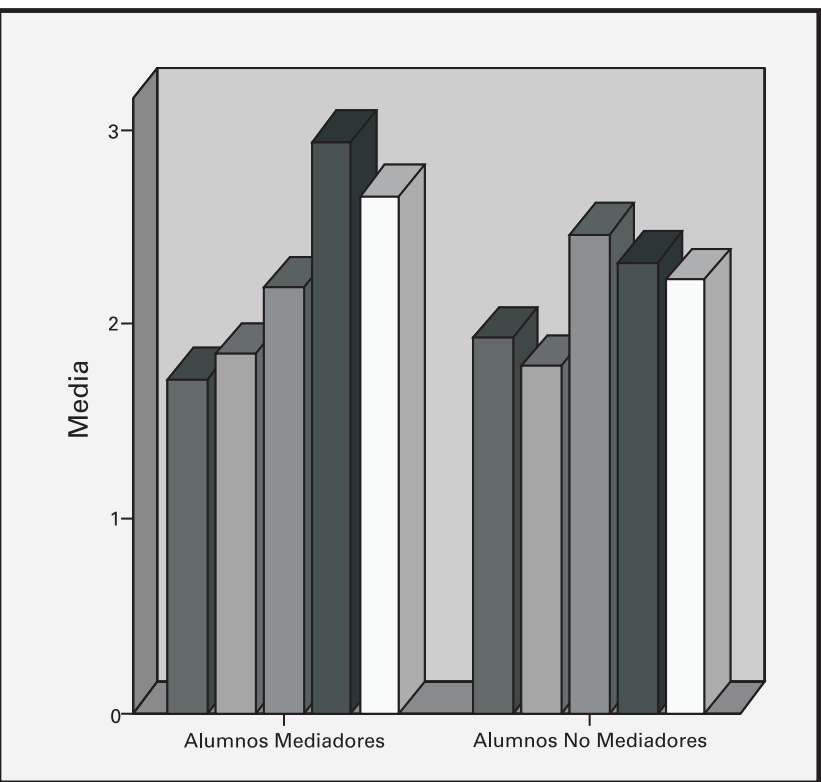

$\begin{array}{ll}\square & \text { Gritar e insultar } \\ \square \quad \text { Pedir Ayuda } \\ \square \quad \text { Ignorar } \\ \square \\ \square \quad \text { Tratar de Entenderlo } \\ \text { Comprenderlo }\end{array}$

Los adolescentes mediadores que han participado en el estudio y tienen preferencia a resolver el conflicto por la vía empática, se benefician de un programa de mediación escolar y han logrado el desarrollo de una de las habilidades sociales mencionadas en nuestra investigación.

Sin embargo, no debemos perder de vista que desde un análisis de frecuencia en la muestra total de estudiantes de educación secundaria, la tendencia a evitar el conflicto, reflejada mediante la opción de ignorar al compañero es del 79\% (véase tabla 2). Y destacamos lo anterior con el fin de hacer mención a que la conducta de evitación del conflicto es una forma susceptible de ser disminuida mediante el desarrollo de habilidades de comunicación, por lo que si creemos en que la mediación escolar

3 Vázquez Gutiérrez, Reyna L. La Mediación Escolar como Herramienta de Educación para la Paz. Tesis Doctoral. Universidad de Murcia. Julio 2012. Gráfica 4. Frecuencia sobre las Formas de Afrontar los Conflictos Agrupación Mediadores y No Mediadores. Pág. 301. 
como programa de educación integral las desarrolla, este tipo de conductas podrán ser disminuidas con el avance del propio programa.

El uso de la mediación potencia las habilidades de comunicación y empatía. La mediación escolar en los centros de educación secundaria, ha logrado cambios positivos en las formas de afrontar el conflicto por los alumnos, permite desarrollar habilidades de comunicación y empatía, en los alumnos mediadores, a diferencia de la tendencia de evitación que muestran los demás alumnos. Creemos que la participación en mediación para la resolución de un conflicto, de igual forma, genera en los alumnos mediados estas habilidades de empatía y diálogo, que permiten afrontar conflictos posteriores con mayor eficacia.

\section{TABLA 3. Frecuencia sobre Formas de Afrontar los Conflictos entre Alumnos. Ignorar $^{4}$}

\begin{tabular}{|ll|r|r|r|r|}
\hline & Frecuencia & Porcentaje & $\begin{array}{c}\text { Porcentaje } \\
\text { válido }\end{array}$ & $\begin{array}{r}\text { Porcentaje } \\
\text { acumulado }\end{array}$ \\
\hline Válidos & Nunca & 25 & 19,4 & 20,0 & 20,0 \\
& Alguna Vez & 53 & 41,1 & 42,4 & 62,4 \\
& Bastantes Veces & 19 & 14,7 & 15,2 & 77,6 \\
& Muchas Veces & 28 & 21,7 & 22,4 & 100,0 \\
Perdidos & Sistema & 125 & 96,9 & 100,0 & \\
Total & 4 & 3,1 & & \\
\hline
\end{tabular}

\section{CONCLUSIONES Y PROPUESTAS}

El éxito de un programa de mediación escolar, en relación con el desarrollo de habilidades sociales en el alumnado, dependerá de la implicación de todos los relacionados con el centro en la búsqueda de un cambio significativo de actitudes y reacciones ante el conflicto, para lograr aprender de cada situación que se presente. Desde su inicio, se encuentra directamente relacionado con el desarrollo de habilidades sociales como la comunicación asertiva, el respeto a lo demás, la convivencia, cooperación y tolerancia, por lo que representa, una herramienta eficaz para lograr los objetivos esenciales de una educación para la paz. Se destaca la necesidad de formar a los estudiantes desde una visión integral de valores y resolución pacífica de los conflictos, que les permita en un futuro adaptarse más fácilmente a los ambientes laborales y sociales de la época moderna. 
Lleva a la práctica elementos tanto de las teorías de la escuela nueva, la educación para la paz, la educación en valores, entre otras propuestas de educación que se han ido convirtiendo en las necesidades de la educación moderna. Logra que los conflictos educativos se conviertan en oportunidades de aprendizaje, tanto para el centro, como para los alumnos que participan en el conflicto. Cuenta con diversas características que la distinguen de los demás programas de convivencia educativa, ya que, impacta directamente con la participación activa del alumnado, del profesorado, y de todos los miembros del centro educativo, además de ser un programa que crece continuamente y con ello asegura a largo plazo el éxito de participación.

Nos parece oportuno retomar en este punto lo expuesto por Aréchaga y Brandoni (2009:37), en relación a que los programas de mediación en la escuela, se basan en objetivos claros, respecto a las normas y habilidades sociales, que se pretenden interiorizar en los alumnos tales como: generar una actitud crítica y reflexiva sobre la modalidad habitual de resolución de conflictos, promover cambios conceptuales y actitudinales en relación con la concepción tradicional y socialmente convalidada del conflicto: ganadores y perdedores, desarrollar en directivos, educadores y estudiantes un sentido de cooperación, mejorar la comunicación entre los miembros de la comunidad educativa, $y$, promover que los estudiantes compartan con los directivos, los docentes y los padres, la responsabilidad de mejorar el ambiente escolar.

El desarrollo de habilidades sociales y de comunicación como la empatía, la capacidad de entender a los demás y su forma de pensar, la asertividad, en beneficio de la construcción correcta de los mensajes de comunicación, la escucha activa, en relación a brindar atención a los demás para entender sus puntos de vista, son elementos directamente relacionados con la búsqueda de una educación integral y la interiorización de valores como la aceptación y el respecto a los demás, además de la resolución pacífica de conflictos.

En nuestra investigación hemos comprobado que un programa de mediación escolar logra desarrollar conductas empáticas en los alumnos involucrados en el mismo, mientras que en un estudio comparativo con alumnos completamente ajenos al programa encontramos que la conducta que con mayor frecuencia muestran ante un conflicto es la de evitar la situación, representada por ignorar al compañero con el que se encuentran involucrados en la problemática.

Encontramos en la mediación escolar, un programa que brinda participación activa a los propios alumnos, una experiencia vivencial, que se aleja de ser un tema o asignatura incluida en el currículo para convertirse en una forma de interacción entre los miembros de la comunidad educativa. Su implementación puede ser dirigida tanto a la prevención como a la disminución de los conflictos escolares. Hemos comprobado que un alumno de educación secundaria que se encuentra involucrado en los programas de mediación escolar es más propenso a utilizar las habilidades sociales de empatía y comunicación para resolver sus conflictos, en comparación con los alum- 
nos ajenos al programa para los cuales es más común reaccionar mediante la evitación del conflicto sin la oportunidad de expresar necesidades y sentimientos.

En un principio, los beneficios sobre las habilidades sociales y de comunicación se interiorizan en los alumnos participantes en el programa, pero es reconocido, que una vez implementado el mismo, los alumnos que hayan sido participes del proceso, como partes en conflicto, valoran esta participación y, mediante la familiarización con el propio programa, podrán ir interiorizando las habilidades a favor de rechazar las formas de enfrentamientos violentos o agresivos.

La mediación escolar, como herramienta de educación para la paz, nos permite tener a la mano un programa en donde son los alumnos los principales protagonistas. La participación se realiza ayudando a los demás compañeros de su edad, allanando así el camino hacia la formación integral en los estudiantes y permitiendo la interiorización de valores cada día más exigidos por las necesidades sociales de nuestra actualidad.

\section{REFERENCIAS BIBLIOGRÁFICAS}

Aréchaga, P. y F. Brandoni. 2009. “La mediación, una contribución a la adquisición de normas sociales en el niño." Pp 36-43 en Mediación escolar. Aportes e interrogantes, resolución de conflictos. La adquisición de normas sociales, editado por F. Brandoni. Argentina: Noveduc Libros.

Asociación Pro Derechos Humanos.1994. Educar para la paz, una propuesta posible. Madrid: Los Libros de Catarata.

Banda, A. 2002. La cultura de la paz. Barcelona: Intermón Oxfam.

Beck, A. T. 2003. Prisioneros del Odio. Las bases de la ira, la hostilidad y la violencia. Barcelona: Paidós.

Boqué Torremorell, M. C. 2010. "Mediación escolar: pasado, presente y futuro." Pp. 209214 en La convivencia escolar: aspectos psicológicos y educativos, editado por J. J. Gazquez y M. del C. Pérez. Granada: GEU.

Brandoni, F. 1999. “Una Introducción a la Mediación Escolar. Dialogos Productivos. Consulta: 7 de Noviembre del 2011 (http://www.dialogosproductivos.net/upload/publications/23022010153000.pdf).

Bush, R., and J. Folger.1996. La promesa de la mediación. Barcelona: Garnica.

Calvo, A. R. 2003. Problemas de convivencia en los centros educativos. Análisis e intervención. Madrid: EOS.

Cano, C., M. P. García-Longoria and E. Ortuño. 2009. Manual de prácticas de mediación escolar. Murcia: Universidad de Murcia.

De la Herrán Gascón, L. 2010. Programa Taldeka para la convivencia escolar. Bilbao: Desclée de Brower.

Fernández, A. 1994. Educando para la Paz: Nuevas Propuestas. Granada: Eirene, Universidad de Granada. 
Fisas, V. 2002. Cultura de Paz y Gestión de los Conflictos. Barcelona: Icaria-Ediciones UNESCO,.

García-Longoria, M.P., y E. Ortuño. 2010. "Aplicación del recurso de la mediación como estrategia de mejora de la convivencia en un centro educativo" Pp. 237-242 en La convivencia escolar: aspectos psicológicos y educativos, de J. J. Gázquez y M. Perez. Granada: GEU.

Germán, A. 2008. "Cultura de paz; nuevas propuestas metodológicas". Pp. 1-3 en Día Internacional de la No Violencia contra la Mujer. Santiago de los Caballeros: Secretaría de Estado de la Mujer.

González, J. and J.B. Martínez. 2007. La violencia en el ámbito escolar. Murcia: Jóvenes Sin Fronteras.

Hareide, D. 2006. Conflict mediation. A nordic perspective. Scandinavian Academic Press.

Jares, X. 1991. Educación para la paz. Su teoría y su prática. Madrid: Popular.

Jares, X. 2006. Pedagogía de la convivencia. Barcelona: Graó.

Lederach, J. P. 1984. Educación para la paz. Objetivo Escolar. Barcelona: Fontamara.

Munné, M. and P. Mac-Cragh. 2006. Los 10 principios de la cultura de mediación. Barcelona: Editorial GRAÓ.

Muñoz, F. 2004. "La paz imperfecta" en Enciclopedia de paz y conflictos, de M. López et al. Granada: Universidad de Granada.

Ortega del Rey, R. 2008. La violencia escolar. Barcelona: Graó.

Pérez, M. J. 2002. "La mediación escolar, proceso de suma de dos modelos de intervención mediadora en la escuela: los programas de mediación escolar y la mediación social intercultural a su paso por instituciones educativas." Educación y futuro: revista de investigación aplicada y experiencias educativas 08: 91-100. Consulta 10 de enero del 2012 (http://dialnet.unirioja.es/servlet/articulo?codigo=2044318).

Pérez-Fuentes, M. C. et. al. 2010. "Programas de mediación educativa" Pp. 269-273 en La convivencia escolar: aspectos psicológicos y educativos, editado por J. J. Gazquez y M. C. Pérez. Granada: GEU.

Rogers, C. 1980. El camino del ser. California: Kairos.

Teixidó, J. 2010. "Se hace camino al andar: sistematización de experiencias de mejora de la convivencia escolar." Pp. 291-298 en La convivencia escolar: aspectos psicológicos y educativos, editado por M. C. Pérez et al. Granada: GEU.

Torrego, J.C. 2000. Mediación de conflictos en instituciones educativas. Manual para la formación de formadores. Madrid: Narcea.

UNESCO. 2002. Mainstreaming the culture of peace. Paris: UNESCO.

Villaescusa, M. 2010. "Condiciones para la mediación de conflictos en Educación Primaria." Pp. 189-192 en La convivencia escolar: aspectos psicológicos y educativos, editado por J. J. Gazquez y M. C. Pérez. Granada: GEU.

Zurbano, J. 1998. Bases de una educación para la paz y la convivencia. Navarra: Gobierno de Navarra. Educación y Cultura. 


\section{comunitania}

REVISTA INTERNACIONAL DE TRABAJO SOCIAL Y CIENCIAS SOCIALES

REVISTA INTERNACIONAL DE TRABAJO SOCIAL Y CIENCIAS SOCIALES
INTERNATIONAL. JOURNAL OF SOCIAL WORK AND SOCIAL SCIENCES

\section{ARTICULOS/ARTICLES}

Estado de bienestar e inversión social en Europa / Welfare State and Social Invesment in Europe

Eguzki Urteaga

Reflexiones sobre el Espacio Público y Participación en las Políticas de Vivienda en Santiago de Chile. Hacia una Práctica Comunitaria / Reflections, on public space and participation in public housinng policies in Santiago de Chile. Towards a community practice

Rodrigo Andrés Mardones

El déficit de transversalidad de género en la Unión Europea / Gender transversality deficit in the European Union

Ángeles Martínez Boyé

Págs 37-60

La construcción de la masculinidad y su relación con la violencia de género / The development of masculinity and relationship to gender violence

$\mathrm{M}^{a}$ Inmaculada López Núñez

Elementos del proceso de mediación que favorecen a su implementación como política social / Elements of the mediation process that benefits implementation as social policy

Paris Alejandro Cabello Tijerina

La mediación escolar y las habilidades sociales en los estudiantes de educación secundaría. Un estudio en institutos de la región de Murcia / School mediation and social skills students in secondary education. A study in institutes of Murcia

María Paz García-Longoria Serrano y Reyna Lizeth Vázquez Gutiérrez

La actuación de los jueces estatales como árbitros privados: un problema de orden público /

State Judge's acting as private arbitrators: a public policy problem

Arnulfo Sánchez García

Págs 137-151

Calidad en los servicios de bienestar en un contexto de crisis económica internacional / Quality in

welfare services in a context of international economic crisis

Eva Sotomayor Morales, Yolanda De la Fuente Robles, Marta García Domingo,

$\mathrm{M}^{\mathrm{a}}$ Luisa Grande Gascón y Tomás Alberich Nistal

Págs 153-179

Contexto de la equidad en los servicios de salud / Context of equity in health services in Mexico

Karla Sáenz López y Delia Téllez Castilla

Los Servicios Sociales en España, puestos a prueba por las personas inmigrantes / Social Services

in Spain, tested by inmigrants

Antonio Gutiérrez Resa

\section{RESEẼAS/REVIEWS}

Rubén Darío Torres Kumbrián. Trabajo Social con Comunidades y Mujeres Musulmanas:

"Diagnóstico de las discriminaciones múltiples" / Social Work with Communities and Muslim women (por Ángeles Martínez Boyé).

Págs 229-234

Manuel Herrera Gómez y José Daniel Barquero Cabrero. Redes sociales: de metáfora a paradigma /

Social Networks: from metaphor to paradigm (por Salvador Gómez García)......

Djamil Tony Kahale Carrillo, Protección a las personas en situación de dependencia / Protection of persons in situations of dependency (por Sara Pérez Martínez). 